\title{
NUV-pumped luminescence of thermally stable samarium-activated alkali metal borophosphate phosphor
}

\author{
Zhenyu FANG, Dan YANG, Youkui ZHENG, Jialiang SONG, \\ Tongsheng YANG, Ruitong SONG, Yuefei XIANG, Jing ZHU* \\ Key Laboratory of LCR Materials and Devices of Yunnan Province, \\ National Center for International Research on Photoelectric and Energy Materials, \\ School of Materials and Energy, Yunnan University, Kunming 650091, China
}

Received: March 2, 2021; Revised: April 28, 2021; Accepted: May 3, 2021

(C) The Author(s) 2021.

\begin{abstract}
Exploring outstanding rare-earth activated inorganic phosphors with good thermostability has always been a research focus for high-power white light-emitting diodes (LEDs). In this study, we report a $\mathrm{Sm}^{3+}$-activated $\mathrm{KNa}_{4} \mathrm{~B}_{2} \mathrm{P}_{3} \mathrm{O}_{13}(\mathrm{KNBP})$ powder phase. Its particle morphology, photoluminescence properties, concentration quenching mechanism, thermal quenching mechanism, and chromatic properties are demonstrated. Upon the near-ultraviolet (NUV) irradiation of $402 \mathrm{~nm}$, the powder phase exhibits orange-red visible luminescence performance, originating from typical ${ }^{4} \mathrm{G}_{5 / 2} \rightarrow{ }^{6} \mathrm{H}_{J / 2}$ $(J=5,7,9)$ transitions of $\mathrm{Sm}^{3+}$. Importantly, the photoluminescence performance has good thermostability, low correlated color temperature (CCT), and high color purity (CP), indicating its promising application in the NUV-pumped warm white LEDs.
\end{abstract}

Keywords: $\mathrm{KNa}_{4} \mathrm{~B}_{2} \mathrm{P}_{3} \mathrm{O}_{13}$ (KNBP); $\mathrm{Sm}^{3+}$ luminescence; concentration quenching; thermostability

\section{Introduction}

To support the field of solid-state lighting and display, developing a variety of inorganic phosphors suitable for white light-emitting diodes (LEDs) has increasingly attracted a tremendous attention [1-3]. However, currently phosphor-converted white LEDs technology is facing the urgent challenge. The increase of operating-temperature generally gives rise to the drop of luminescence efficiency of phosphor due to the long-time work of white LEDs. In addition, excellent red phosphor is urgently needed for reducing correlated color temperature and improving color rending index

\footnotetext{
* Corresponding author.

E-mail: jzhu@ynu.edu.cn
}

of white LEDs. Hence, constructing red phosphor with high thermal stability becomes a research focus in phosphor-converted white LED technology [4,5].

Generally, an LED inorganic phosphor is composed of activator and host material. The activators are doped into a host material to produce luminescence properties. Rare-earth ions, as important activators, can acquire easily various emission light colors (blue, green, yellow, and red) owing to their abundant $4 \mathrm{f}$ transition properties [6,7]. To cope with the needs of white LEDs for red phosphors, inorganic aluminates [8,9], phosphates $[10,11]$, borates [12], silicate [13], tungstate [14], vanadate [15], and molybdate [16] activated with rare earth activator samarium (Sm) were reported. The typical near-ultraviolet (NUV) $4 \mathrm{f}$ absorption of $\mathrm{Sm}^{3+}$ matches well with the emission of NUV LED chips. 
The $4 \mathrm{f}$ transitions of $\mathrm{Sm}^{3+}$ dominate its visible light emission. The symmetry of the coordination field for $\mathrm{Sm}^{3+}$ ions closely affects the luminescence efficiency and emission light color of inorganic phosphors.

Borophosphate materials have been proven to have good optical, physical, and chemical properties correlated with the diversified anionic frameworks, which originate from the various connections of boron-oxygen tetrahedron $\left(\mathrm{BO}_{4}\right)$, boron-oxygen triangle $\left(\mathrm{BO}_{3}\right)$, and phosphorus-oxygen tetrahedron $\left(\mathrm{PO}_{4}\right)$ structural groups [17]. So, the diversified structures of borophosphate materials give a great probability for exhibiting the different emission light colors of $\mathrm{Sm}^{3+}$ related to the diversity of its coordination fields. In 2019, Yang et al. [18] firstly reported borophosphate $\mathrm{KNa}_{4} \mathrm{~B}_{2} \mathrm{P}_{3} \mathrm{O}_{13}$ (KNBP) with the non-centrosymmetric $P n a 2_{1}$ space group and its nonlinear optical properties. The $\left(\mathrm{B}_{2} \mathrm{P}_{3} \mathrm{O}_{13}\right)^{5-}$ anionic framework of the borophosphate is composed of $\mathrm{BO}_{4}$ and $\mathrm{PO}_{4}$ tetrahedra. The borophosphate KNBP exhibits a good transmittance in the whole visible light region. Besides, it has the characteristics of stable physicochemical properties, facile preparation, and nontoxicity. The previous investigation indicates that the inorganic KNBP borophosphate could be a prospective host of phosphor materials. However, KNBP-based phosphor doped with $\mathrm{Sm}^{3+}$ activators has not been proposed.

In this work, we utilized a facile solid-state synthesis procedure for synthesizing KNBP: $x \mathrm{Sm}^{3+}(x=$ $0,0.25,0.5,0.75,1,2,3 \mathrm{~mol} \%$ ) powder phases. The phase identification, morphological characterization, photoluminescence properties, chromatic properties, concentration quenching, and thermal quenching mechanisms were systematically analyzed.

\section{Experimental}

\section{1 Material synthesis}

The reagents used in the experiment were $\mathrm{Sm}_{2} \mathrm{O}_{3}$ (99.99\%), $\mathrm{H}_{3} \mathrm{BO}_{3}$ (99.9\%), $\mathrm{NH}_{4} \mathrm{H}_{2} \mathrm{PO}_{4}(99.9 \%), \mathrm{K}_{2} \mathrm{CO}_{3}$ (99.9\%), and $\mathrm{Na}_{2} \mathrm{CO}_{3}(99.9 \%)$. According to the stoichiometric ratio of the KNBP host, the required reagents were weighed precisely. After mixing and grinding these reagents, the mixture was preheated at $673 \mathrm{~K}$ for $5 \mathrm{~h}$ in a high-temperature furnace. Then the temperature was increased to $873 \mathrm{~K}$. The heat preservation period was $168 \mathrm{~h}$ in Ref. [18]. To optimize the synthesis procedure, we carried out a series of solid-state reactions with different heat preservation periods $(24,48,72,120$, and $168 \mathrm{~h})$. The sintering time-dependent X-ray diffraction (XRD) patterns of the KNBP host powder phase are shown in Fig. S1 in the Electronic Supplementary Material (ESM). The pure KNBP powder with good crystallinity can be prepared in only $24 \mathrm{~h}$. Thus, a series of KNBP: $x \mathrm{Sm}^{3+}(x=0$, $0.25,0.5,0.75,1,2,3 \mathrm{~mol} \%$ ) polycrystalline phases were synthesized by the more simplified solid-state synthesis procedure, in comparison to the preparation reported in Ref. [18].

\section{2 Characterizations}

Powder XRD patterns were recorded via a Rigaku D/Max-3B diffractometer with $\mathrm{Cu} \mathrm{K \alpha}$ radiation. A Nicolet iS10 spectrometer was used for recording Fourier transform infrared (FTIR) spectra. The morphology observation was carried out via a scanning electron microscope (SEM, FEI QUANTA200) and a transmission electron microscope (TEM, JEOL 2100F), and the elemental analysis was made by the scanning electron microscope with an energy dispersive spectroscopy (EDS) detector. The UV-Vis diffuse reflectance spectra (DRS) were performed via a SHIMADZU UV2600 spectrophotometer. A Hitachi F7100 spectrophotometer equipped with an integrating sphere was used to measure photoluminescence excitation (PLE), photoluminescence (PL), and internal quantum efficiency (IQE). An Edinburgh FLS920 spectrophotometer with xenon lamp excitation was employed to measure decay lifetime.

\section{Results and discussion}

\section{1 XRD and FTIR analysis}

The powder XRD reflections for the prepared KNBP: $x \mathrm{Sm}^{3+}(x=0,0.25,0.5,0.75,1,2,3 \mathrm{~mol} \%)$ phases are displayed in Fig. 1(a). Based on the crystallographic information file (CIF) of the KNBP orthorhombic phase reported in 2019 [18], we have used the Diamond software to simulate its XRD reflection. The XRD patterns for all $\mathrm{Sm}^{3+}$-doped samples have the same peak positions and relative intensities as the simulated one, revealing that all the prepared powder phases are isostructural with the KNBP orthorhombic matrix and belong to the non-centrosymmetric space group $P n a 2_{1}$. There are crystallographically independent four sodium (Na), 
one potassium (K), two boron (B), three phosphorus $(\mathrm{P})$, and thirteen oxygen $(\mathrm{O})$ atoms in its asymmetric unit. $\mathrm{K}, \mathrm{B}$, and $\mathrm{P}$ atoms are connected with eight, four, and four $\mathrm{O}$ atoms, respectively. Figure 1(b) shows the 5,6 , and 10-coordinated environments of $\mathrm{Na}$ atoms. The coordination number $(\mathrm{CN})$-dependent radius values for $\mathrm{K}^{+}, \mathrm{Na}^{+}, \mathrm{B}^{3+}, \mathrm{P}^{5+}$, and $\mathrm{Sm}^{3+}$ ions are listed in Table S1 in the ESM [19]. There is a good approximation for the ionic radius values of $\mathrm{Na}^{+}$and $\mathrm{Sm}^{3+}$ ions. The dopant $\mathrm{Sm}^{3+}$ ion prefers to occupy the lattice position of the $\mathrm{Na}^{+}$ion, and the structure of the KNBP host is not altered. To discuss the influence of the dopant $\mathrm{Sm}^{3+}$ ion on the shift of diffraction peak, the enlarged XRD patterns in the $29^{\circ}-30.5^{\circ}$ range are shown in Fig. 1(a). The strongest peak at around $29.7^{\circ}$ slightly shifts toward a higher $2 \theta$ angle with the increase of the dopant $\mathrm{Sm}^{3+}$ concentration, supporting the fact that $\mathrm{Sm}^{3+}$ ion is doped into the host lattice and substitute $\mathrm{Na}^{+}$ion. The main reason for the peak-shift phenomenon is that the radius of the dopant $\mathrm{Sm}^{3+}$ ion is slightly smaller than that of the substituted $\mathrm{Na}^{+}$ion (Table S1 in the ESM). However, increasing the dopant concentration of $\mathrm{Sm}^{3+}$ ions to $3 \mathrm{~mol} \%$ gives rise to a weak diffraction peak at $2 \theta=31.7^{\circ}$, corresponding to a small amount of impurity phase $\mathrm{KBO}_{2}$ (JCPDS 03-0729) due to the non-equivalent replacement of $\mathrm{Sm}^{3+}$ for $\mathrm{Na}^{+}$and the solid solubility limitation, which also resulted in the appearance of the second phase in $\mathrm{Ba}_{3}\left(\mathrm{VO}_{4}\right)_{2}: \mathrm{Sm}^{3+}$ and $\mathrm{LiNa}_{2} \mathrm{~B}_{5} \mathrm{P}_{2} \mathrm{O}_{14}: \mathrm{Eu}^{3+}$ phosphors reported recently $[15,20]$. Besides, to verify the purity of the as-synthesized samples, the XRD data of the KNBP phase and KNBP: $0.75 \% \mathrm{Sm}^{3+}$ powder was refined by the Rietveld method, as shown in Fig. 2. Here, the basis refinement was derived from the KNBP initial structure parameters (orthorhombic system with space group $\left.P n a 2_{1}\right)$. The refinement results of profile residual factor $\left(R_{\mathrm{p}}\right)$ and weighted profile $R$-factor $\left(R_{\mathrm{wp}}\right)$ are reliable and reasonable owing to $R_{\mathrm{p}}=7.42 \%, R_{\mathrm{wp}}=$
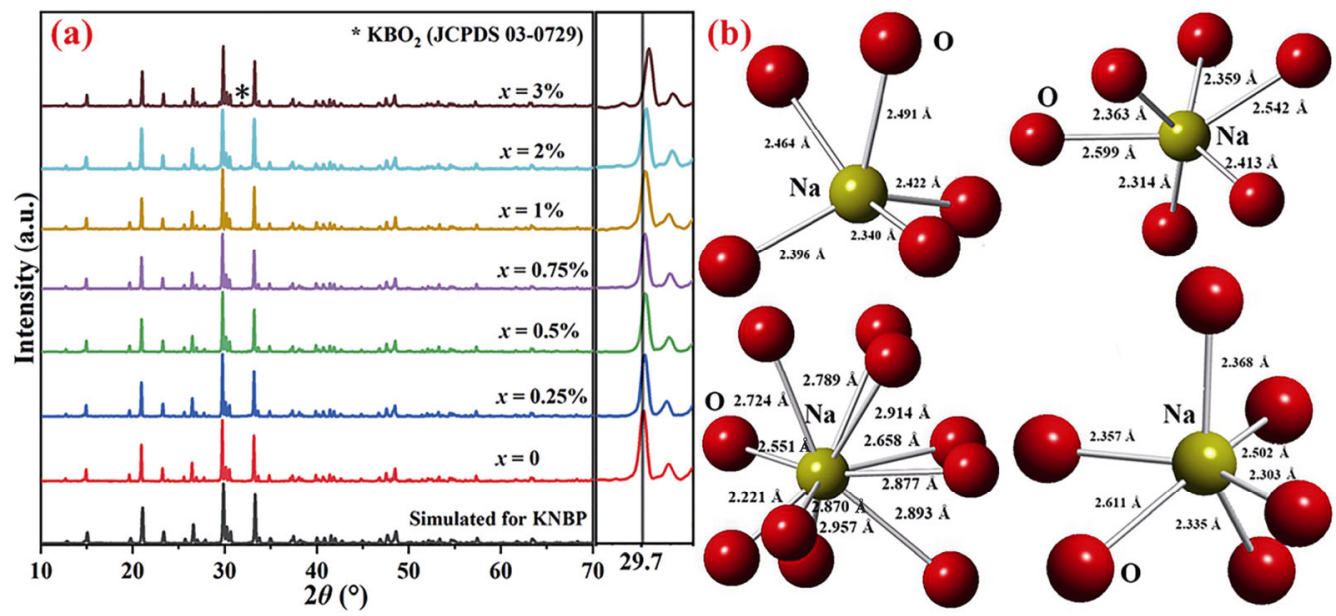

Fig. 1 (a) Powder XRD patterns of the prepared KNBP: $x \mathrm{Sm}^{3+}(x=0,0.25,0.5,0.75,1,2,3$ mol\%) phases and (b) coordination environments for $\mathrm{Na}$ atoms.
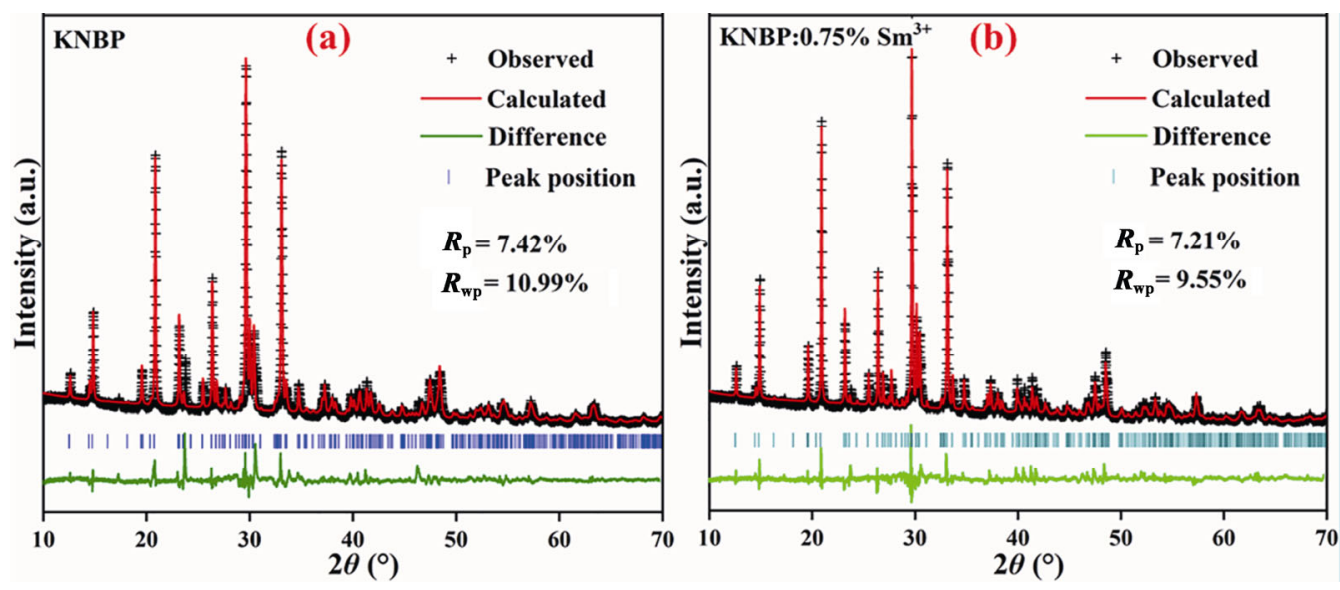

Fig. 2 Rietveld refinements of XRD patterns of the (a) KNBP phase and (b) KNBP: $0.75 \% \mathrm{Sm}^{3+}$ powder. 
$10.99 \%$ for the KNBP phase and $R_{\mathrm{p}}=7.21 \%, R_{\mathrm{wp}}=$ $9.55 \%$ for the KNBP: $0.75 \% \mathrm{Sm}^{3+}$ powder, indicating that the samples have good phase purity.

Figure 3 displays the FTIR curves of the KNBP: $x \mathrm{Sm}^{3+}$ $(x=0,0.25,0.5,0.75,1,2,3 \mathrm{~mol} \%)$ powders. All the FTIR spectra with dopant $\mathrm{Sm}^{3+}$ ions keep the same shapes and locations of vibration peaks as that of the pure KNBP phase. These absorption vibrations are caused by $\mathrm{BO}_{4}$ and $\mathrm{PO}_{4}$ tetrahedra [21,22], which are basic structural groups in the KNBP matrix. The vibrations at 1193 and $1148 \mathrm{~cm}^{-1}$ result from the asymmetric stretching mode of the $\mathrm{PO}_{4}$ group. The vibrations at 1093 and $918 \mathrm{~cm}^{-1}$ originate from the symmetric stretching mode of the $\mathrm{BO}_{4}$ group. The vibration at $986 \mathrm{~cm}^{-1}$ arises from the $\mathrm{P}-\mathrm{O}-\mathrm{P}$ asymmetric stretching. The vibrations at 869 and $821 \mathrm{~cm}^{-1}$ are ascribed to the B-O-P asymmetric stretching. The vibrations at 750 and $674 \mathrm{~cm}^{-1}$ correspond to the symmetric stretching of $\mathrm{B}-\mathrm{O}-\mathrm{P}$ and $\mathrm{P}-\mathrm{O}-\mathrm{P}$. The vibrations at 622 and $591 \mathrm{~cm}^{-1}$ belong to the asymmetric bending of the $\mathrm{BO}_{4}$ group. The vibration at $542 \mathrm{~cm}^{-1}$ is assigned to the asymmetric bending of the $\mathrm{PO}_{4}$ group. The symmetric bending of the $\mathrm{BO}_{4}$ and $\mathrm{PO}_{4}$ groups result in the vibrations at 521 and $468 \mathrm{~cm}^{-1}$. The investigation confirms that the doping of $\mathrm{Sm}^{3+}$ ions does not destroy the $\mathrm{BO}_{4}$ and $\mathrm{PO}_{4}$ structural units.

\section{2 Particle morphology and elemental analysis}

Particle morphology and size of a phosphor is closely related to photoluminescence performance. The comparative investigation on the particle morphologies of the KNBP host and KNBP: $0.75 \% \mathrm{Sm}^{3+}$ powder was carried out (Figs. 4(a) and 4(b)). Both powder samples

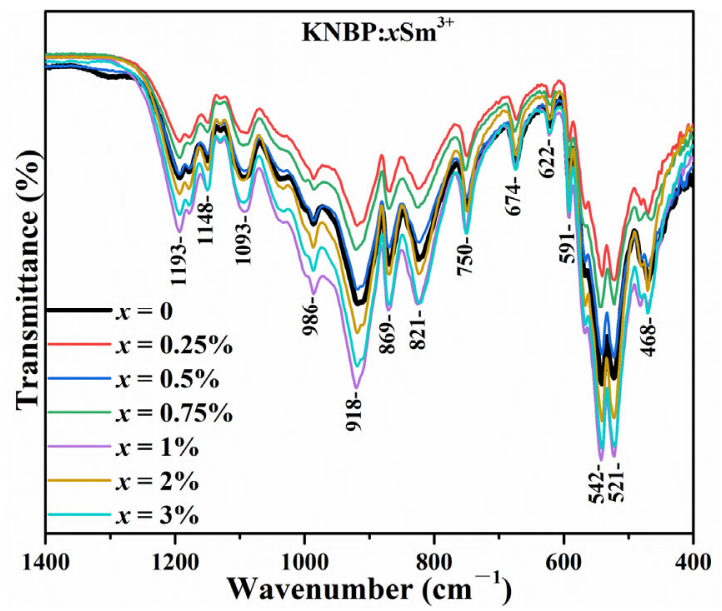

Fig. 3 FTIR spectra of KNBP: $x \mathrm{Sm}^{3+}(x=0,0.25,0.5$, $0.75,1,2,3 \mathrm{~mol} \%$ ) powders. consist of block-like micron particles with irregular shape and inhomogeneous size. During the hightemperature sintering process, adjacent small-sized particles have aggregated to attain micron bulks. The aggregation of the KNBP: $0.75 \% \mathrm{Sm}^{3+}$ particles (Fig. 4(b)) is more serious than that of the KNBP particles (Fig. 4(a)). The TEM image of the KNBP: $0.75 \% \mathrm{Sm}^{3+}$ powder (Fig. 4(c)) shows single particle with a micronlevel size. The inset of Fig. 4(c) shows the corresponding high-resolution TEM (HRTEM) image. The lattice fringe with $0.424 \mathrm{~nm}$ corresponds to the (112) crystal plane of the host phase. The clear lattice fringe verifies that the sample has good crystallization and stability under high energy electron beam. Figure 4(d) depicts the measured EDS profile of the KNBP: $0.75 \% \mathrm{Sm}^{3+}$ sample, which manifests the presence of the elements $\mathrm{Sm}, \mathrm{K}, \mathrm{Na}, \mathrm{B}, \mathrm{P}$, and $\mathrm{O}$. The elemental distribution map for the KNBP: $0.75 \% \mathrm{Sm}^{3+}$ sample is shown in Fig. 4(e), confirming the uniform distribution of these chemical components.

\section{3 Diffuse reflection, excitation, and emission}

The measured DRS curves of the KNBP: $x \mathrm{Sm}^{3+}(x=0$, $0.25,0.5,0.75,1,2,3 \mathrm{~mol} \%$ ) powder phases at room temperature are presented in Fig. 5. The KNBP matrix has no absorption in the visible (Vis) region, which keeps consistent with the diffuse reflection data reported by Yang et al. [18]. The observed ultraviolet (UV) absorption mainly comes from the charge transitions of $\mathrm{BO}_{4}$ and $\mathrm{PO}_{4}$ groups [18]. Differently, as depicted in the inset of Fig. 5, all the DRS curves with dopant $\mathrm{Sm}^{3+}$ ions clearly show UV-Vis absorptions in the $330-510 \mathrm{~nm}$ range owing to the typical $4 \mathrm{f}-4 \mathrm{f}$ transitions of $\mathrm{Sm}^{3+}$. A series of absorptions appear at $344,361,374,402,438$, and $476 \mathrm{~nm}$, deriving from the ${ }^{6} \mathrm{H}_{5 / 2} \rightarrow{ }^{4} \mathrm{H}_{9 / 2},{ }^{4} \mathrm{D}_{3 / 2},{ }^{4} \mathrm{D}_{1 / 2},{ }^{4} \mathrm{~F}_{7 / 2},{ }^{4} \mathrm{G}_{9 / 2}$, and ${ }^{4} \mathrm{G}_{7 / 2}$ transitions, respectively [23]. Among these absorptions, the ${ }^{6} \mathrm{H}_{5 / 2} \rightarrow{ }^{4} \mathrm{~F}_{7 / 2}(402 \mathrm{~nm})$ transition is the strongest.

The PLE curve of the KNBP: $0.75 \% \mathrm{Sm}^{3+}$ powder recorded at the monitoring wavelength of $598 \mathrm{~nm}$ is depicted in Fig. 6(a). We can observe excitation peaks at $344\left({ }^{6} \mathrm{H}_{5 / 2} \rightarrow{ }^{4} \mathrm{H}_{9 / 2}\right), 361\left({ }^{6} \mathrm{H}_{5 / 2} \rightarrow{ }^{4} \mathrm{D}_{3 / 2}\right), 374\left({ }^{6} \mathrm{H}_{5 / 2} \rightarrow\right.$ $\left.{ }^{4} \mathrm{D}_{1 / 2}\right), 402\left({ }^{6} \mathrm{H}_{5 / 2} \rightarrow{ }^{4} \mathrm{~F}_{7 / 2}\right), 415\left({ }^{6} \mathrm{H}_{5 / 2} \rightarrow{ }^{6} \mathrm{P}_{5 / 2}\right), 438\left({ }^{6} \mathrm{H}_{5 / 2} \rightarrow\right.$ $\left.{ }^{4} \mathrm{G}_{9 / 2}\right)$, and $469 \mathrm{~nm}\left({ }^{6} \mathrm{H}_{5 / 2} \rightarrow{ }^{4} \mathrm{I}_{13 / 2}\right)$ [24]. The strongest transition appears at $402 \mathrm{~nm}\left({ }^{6} \mathrm{H}_{5 / 2} \rightarrow{ }^{4} \mathrm{~F}_{7 / 2}\right)$, which keeps consistent with the obtained diffuse reflectance result in Fig. 5. The comparison of photoluminescence under various excitations was investigated. Figure 6(b) illustrates the PL curves of the KNBP:0.75\% $\mathrm{Sm}^{3+}$ 

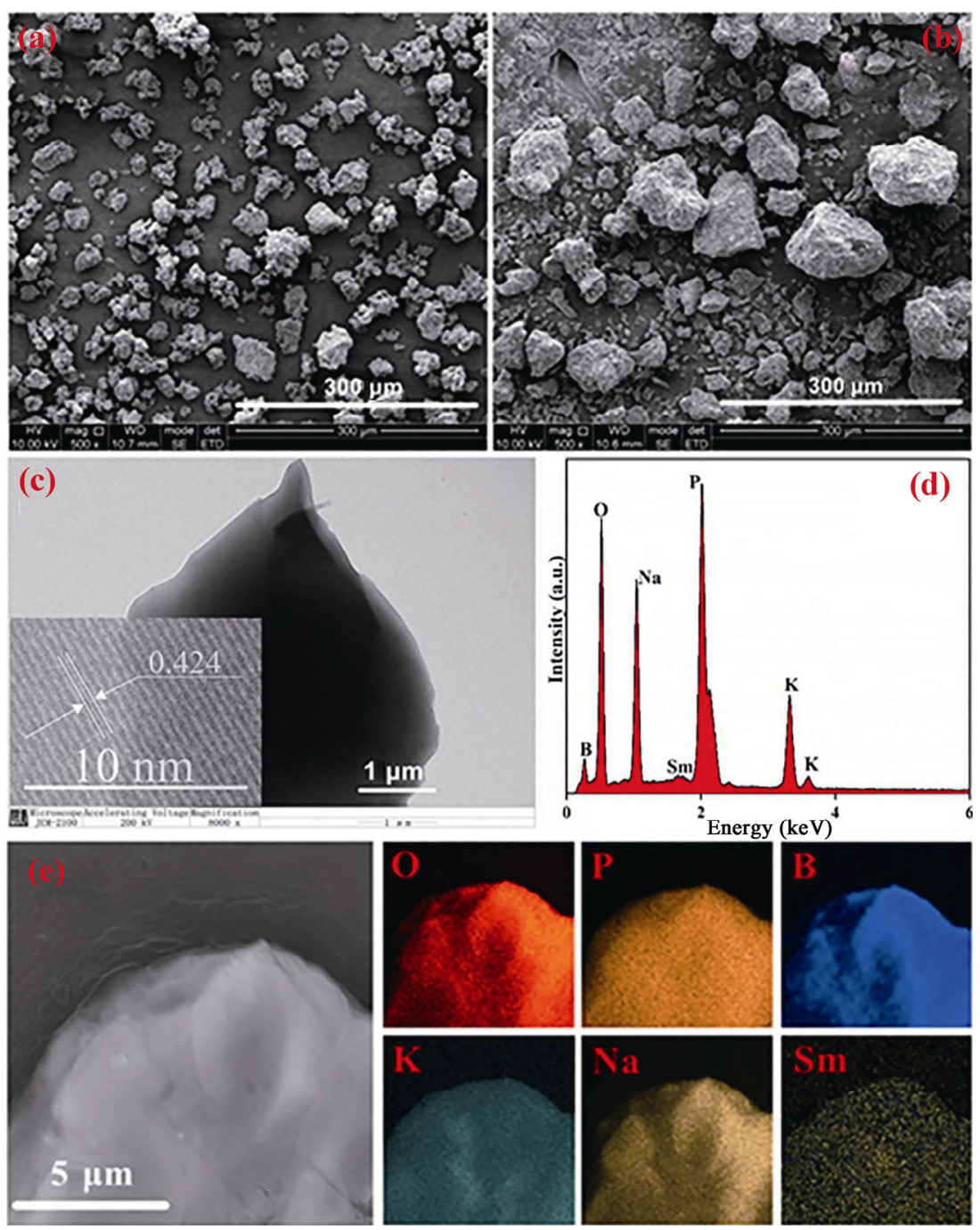

Fig. 4 (a) SEM image (scale bar $=300 \mu \mathrm{m}$ ) of the KNBP phase; (b) SEM image (scale bar $=300 \mu \mathrm{m}$ ); (c) TEM image (scale bar $=1 \mu \mathrm{m}$ ), the inset shows the HRTEM image; (d) EDS spectrum; and (e) elemental mappings of the KNBP:0.75\% $\mathrm{Sm}^{3+}$ powder.

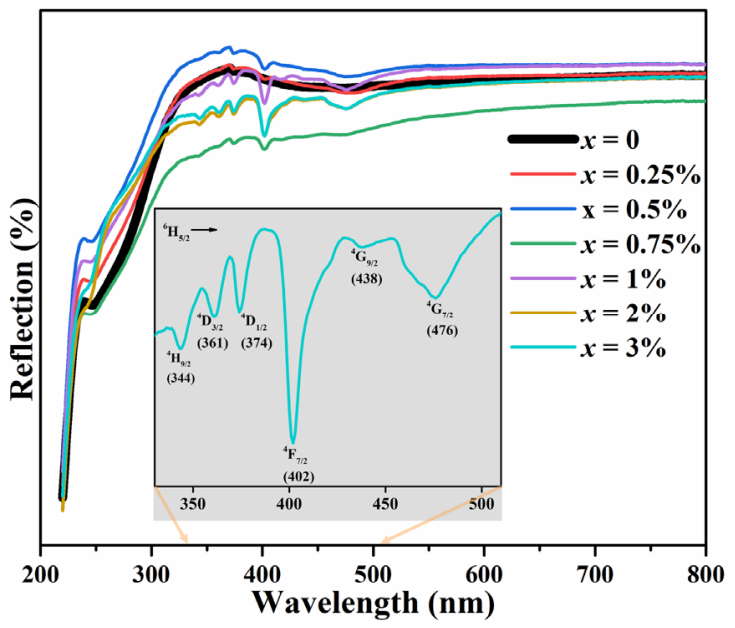

Fig. 5 DRS curves of the prepared KNBP: $x \mathrm{Sm}^{3+}(x=0$, $0.25,0.5,0.75,1,2,3 \mathrm{~mol} \%)$ powder phases (the inset shows the enlarged curve in the $330-510 \mathrm{~nm}$ range). powder upon excitations at $344,361,374,402,415$, and $469 \mathrm{~nm}$. All the PL curves are mainly composed of three typical $4 \mathrm{f}-4 \mathrm{f}$ transitions of $\mathrm{Sm}^{3+}$, which are located at $645 \mathrm{~nm}\left({ }^{4} \mathrm{G}_{5 / 2} \rightarrow{ }^{6} \mathrm{H}_{9 / 2}\right), 598\left({ }^{4} \mathrm{G}_{5 / 2} \rightarrow{ }^{6} \mathrm{H}_{7 / 2}\right)$, and $562\left({ }^{4} \mathrm{G}_{5 / 2} \rightarrow{ }^{6} \mathrm{H}_{5 / 2}\right)$ [16]. The dominant ${ }^{4} \mathrm{G}_{5 / 2} \rightarrow{ }^{6} \mathrm{H}_{7 / 2}$ $(598 \mathrm{~nm})$ transition belongs to visible orange-red light. Differently, the integrated emission intensity obtained by $402 \mathrm{~nm}$ excitation is higher than others, indicating that an NUV LED chip is suitable for NUV-pumped photoluminescence of the sample. As a rule, the purely magnetic dipole transition (MDT) and purely electric dipole transition (EDT) are known as ${ }^{4} \mathrm{G}_{5 / 2} \rightarrow{ }^{6} \mathrm{H}_{5 / 2}$ and ${ }^{4} \mathrm{G}_{5 / 2} \rightarrow{ }^{6} \mathrm{H}_{9 / 2}$ transition in the same order. The combination of MDT and EDT results in the ${ }^{4} \mathrm{G}_{5 / 2} \rightarrow{ }^{6} \mathrm{H}_{7 / 2}$ transition. The asymmetry of the local coordination of $\mathrm{Sm}^{3+}$ can be used to analyze the EDT/MDT intensity ratio. The 

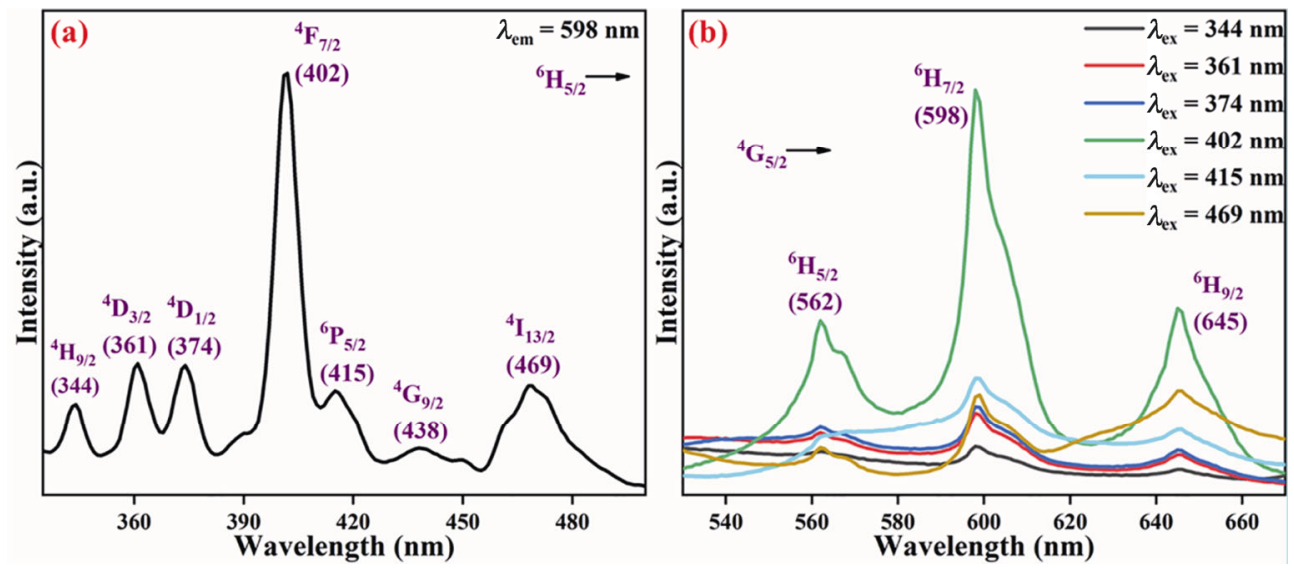

Fig. 6 (a) PLE and (b) PL curves of the KNBP:0.75\% $\mathrm{Sm}^{3+}$ powder.

asymmetric coordination of $\mathrm{Sm}^{3+}$ corresponds to the intensity ratio of EDT/MDT $>1$. In this case, the coordination environment of $\mathrm{Na}^{+}$is distorted (Fig. 1(b)). The local coordination of $\mathrm{Sm}^{3+}$ has a slight asymmetry due to the substitution of $\mathrm{Sm}^{3+}$ for $\mathrm{Na}^{+}$. Hence, the MDT intensity is slightly less than the EDT one (Fig. 6(b)) [15].

\section{3. $4 \mathrm{Sm}^{3+}$ concentration-dependent photoluminescence}

Figure 7(a) shows the PL spectra for the KNBP: $x \mathrm{Sm}^{3+}$ $(x=0.25,0.5,0.75,1,2,3 \mathrm{~mol} \%)$ powder samples upon $402 \mathrm{~nm}$ NUV excitation. The $\mathrm{Sm}^{3+}$ doping concentration closely affects emission intensity. Figure 7(b) illustrates the $\mathrm{Sm}^{3+}$ concentration-dependent emission intensity. As the $\mathrm{Sm}^{3+}$ concentration increases from 0.25 to $0.75 \mathrm{~mol} \%$, the PL intensity increases. Subsequently, the non-radiative energy transfer among $\mathrm{Sm}^{3+}$ ions occurs with the further increase of the $\mathrm{Sm}^{3+}$ concentration, leading to the decrease of the emission intensity. The $\mathrm{Sm}^{3+}$ concentration of $0.75 \mathrm{~mol} \%$ is determined to be the optimization for concentration quenching. As a rule, the critical energy transfer distance
$\left(R_{\mathrm{c}}\right)$ for concentration quenching is greater than $5 \AA$, meaning that the multipole-multipole interaction is the cause of concentration quenching. In order to discuss the mechanism of concentration quenching, the $R_{\mathrm{c}}$ value of $\mathrm{Sm}^{3+}$ in the KNBP matrix could be estimated using Eq. (S1) in the ESM. The calculated $R_{\mathrm{c}}$ value of $\mathrm{Sm}^{3+}$ is $26.1 \AA$. Hence, the concentration quenching for KNBP: $x \mathrm{Sm}^{3+}$ comes from the multipole-multipole interaction. The multipolar energy transfer process was further analyzed via Eqs. (S2) and (S3) in the ESM. The obtained result is illustrated in Fig. 7(c), which stands for the linear correlation of $\log (I / \chi)$ versus $\log (\chi)$. On the basis of the slope $-\theta / 3=-1.73$, the obtained $\theta$ value equals 5.19 , which is near to 6 . The result manifests that the electric dipole-dipole interaction dominates the concentration quenching of the prepared KNBP: $x \mathrm{Sm}^{3+}$ powder samples.

Figure 8 shows the lifetime for the KNBP: $x \mathrm{Sm}^{3+}$ $(x=0.25,0.5,0.75,1,2,3 \mathrm{~mol} \%)$ powder samples, which were recorded under ${ }^{6} \mathrm{H}_{5 / 2} \rightarrow{ }^{4} \mathrm{~F}_{7 / 2}(402 \mathrm{~nm})$ excitation and ${ }^{4} \mathrm{G}_{5 / 2} \rightarrow{ }^{6} \mathrm{H}_{7 / 2}(598 \mathrm{~nm})$ emission. All decay lifetime curves were fitted using a typical double exponential
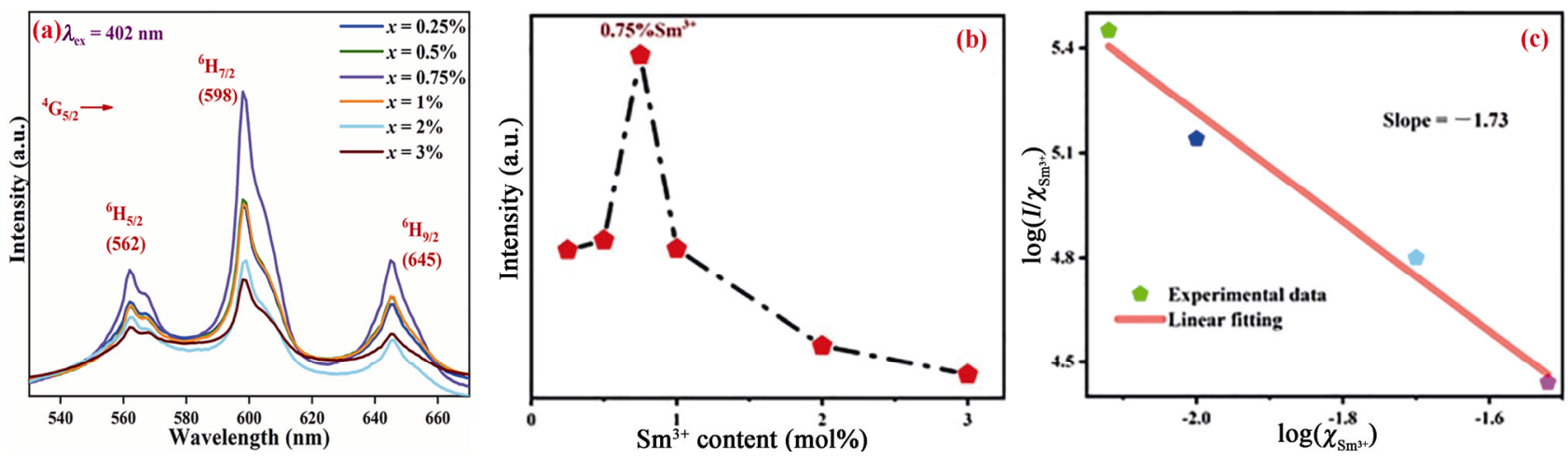

Fig. 7 (a) PL curves, (b) $\mathrm{Sm}^{3+}$ concentration-dependent PL intensity, and (c) dependence of $\log (I / \chi)$ on $\log (\chi)$ for KNBP: $x \mathrm{Sm}{ }^{3+}$ $(x=0.25,0.5,0.75,1,2,3 \mathrm{~mol} \%)$. 

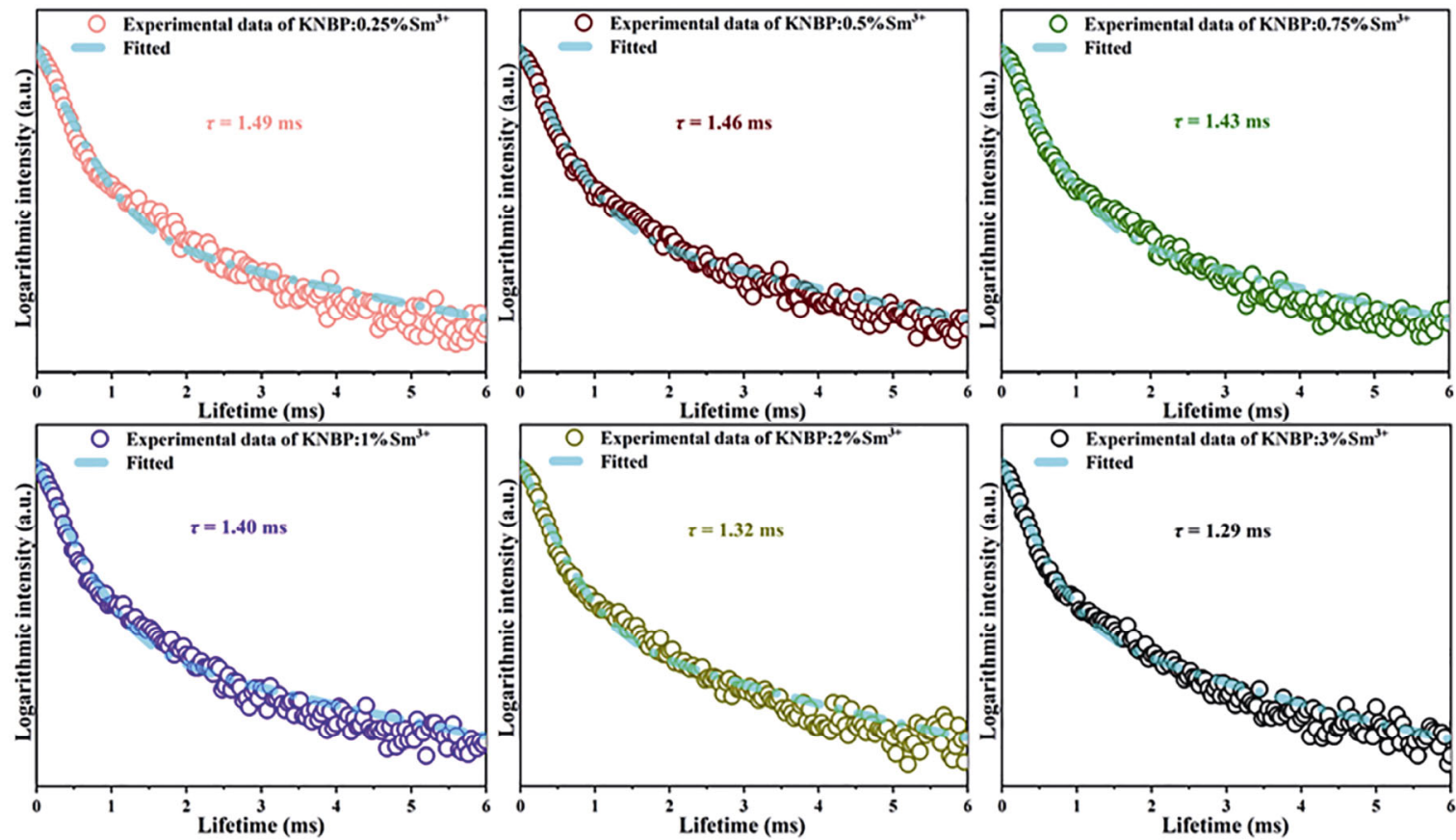

Fig. 8 Decay lifetime curves for KNBP: $x \mathrm{Sm}^{3+}(x=0.25,0.5,0.75,1,2,3 \mathrm{~mol} \%)$.

function, which is shown as Eq. (S4) in the ESM. The average decay lifetime $\left(\tau_{\text {ave }}\right)$ was calculated via Eq. (S5) in the ESM. As the $\mathrm{Sm}^{3+}$ doping concentration increases, the $\tau_{\text {ave }}$ value shortens from 1.49 to $1.29 \mathrm{~ms}$. The sample with the optimized doping concentration of $0.75 \mathrm{~mol} \%$ possesses the $\tau_{\text {ave }}$ value of $1.43 \mathrm{~ms}$. The downtrend of decay lifetime arises from the increased probability of the non-radiative transitions among dopant $\mathrm{Sm}^{3+}$ ions $[25,26]$.

Figure 9(a) shows the CIE chromaticity diagram for the KNBP: $x \mathrm{Sm}^{3+}(x=0.25,0.5,0.75,1,2,3 \mathrm{~mol} \%)$ powder samples. The inset of Fig. 9(a) lists the values of color coordinates $(x, y)$, correlated color temperature
(CCT), and color purity (CP), which were calculated using Eqs. (S6)-(S9) in the ESM, respectively. The emission colors of all the samples upon $402 \mathrm{~nm}$ NUV irradiation are situated in the orange-red area. The CCT parameters range from 2050 to $2890 \mathrm{~K}$, which are far lower than $5000 \mathrm{~K}$. The low CCT indicates the potential application of the title phosphor in warm white LEDs. Furthermore, the CP values of all the samples are higher than $80 \%$. Figure 9 (b) illustrates the comparison of the digital photos of the KNBP: $0.75 \% \mathrm{Sm}^{3+}$ powder under daylight and a $365 \mathrm{~nm} \mathrm{UV}$ lamp. When the KNBP: $0.75 \% \mathrm{Sm}^{3+}$ white powder was excited by a $365 \mathrm{~nm}$ UV lamp, the obtained emission
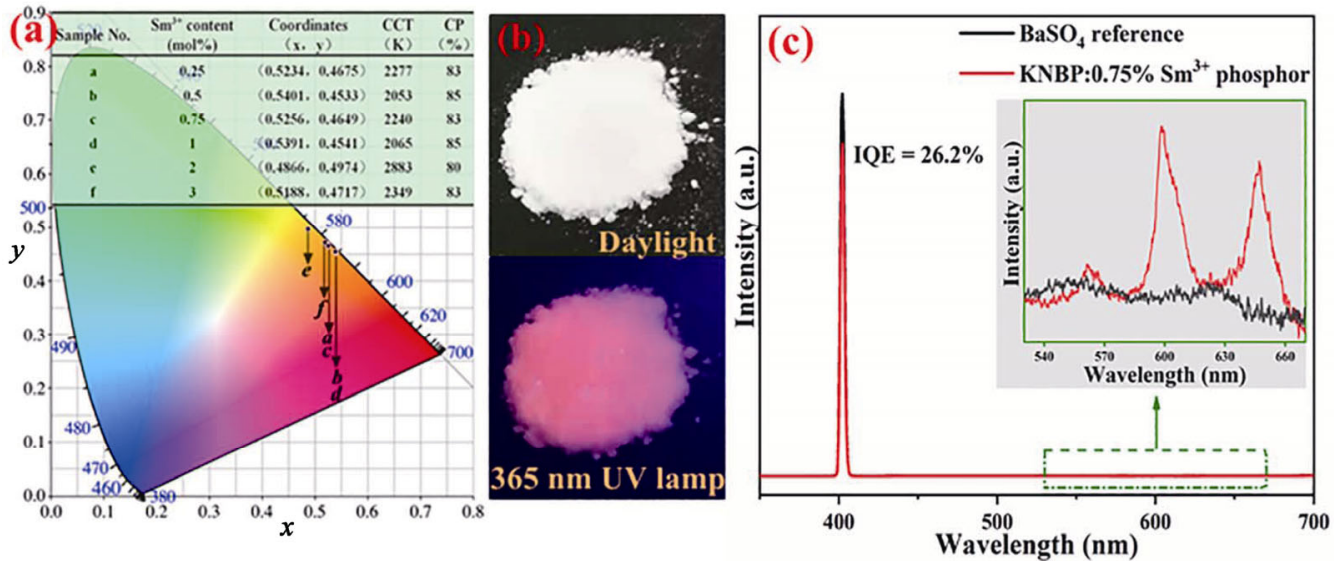

Fig. 9 (a) CIE chromaticity diagram for KNBP: $x \mathrm{Sm}^{3+}(x=0.25,0.5,0.75,1,2,3 \mathrm{~mol} \%)$ (the inset shows the CIE coordinates, $\mathrm{CCT}$, and CP values), (b) comparison on the digital photos of the KNBP:0.75\% $\mathrm{Sm}^{3+}$ powder under daylight and a $365 \mathrm{~nm} \mathrm{UV}$ lamp, and (c) the IQE measurement of the KNBP: $0.75 \% \mathrm{Sm}^{3+}$ powder. 
color is comparable to the above color coordinate data. Besides, Eq. (S10) in the ESM was employed for assessing the IQE value. Under $402 \mathrm{~nm}$ excitation, the IQE value of the KNBP: $0.75 \% \mathrm{Sm}^{3+}$ powder is $26.2 \%$, as depicted in Fig. 9(c).

\section{5 Operating temperature-dependent photolumi- nescence}

Figure 10(a) illustrates the correlation between operating temperature and PL intensity for the KNBP: $0.75 \% \mathrm{Sm}^{3+}$ powder upon $402 \mathrm{~nm}$ NUV irradiation. As the operating temperature increases to $528 \mathrm{~K}$, there is no obvious change in the positions and peak shapes for the $4 \mathrm{f}-4 \mathrm{f}$ transitions of $\mathrm{Sm}^{3+}$. However, the integrated PL intensity slowly drops due to the increased non-radiative transitions stimulated at high temperatures. Figure 10(b) illustrates clearly the normalized emission intensities depending on different temperatures. When the operating temperature rises to $428 \mathrm{~K}$, the loss of the integrated PL intensity is $15 \%$ of the initial intensity, which is comparable with the $14 \%$ loss of the commercially available phosphor $\mathrm{Sr}_{2} \mathrm{Si}_{5} \mathrm{~N}_{8}: \mathrm{Eu}^{2+}$ [4]. To further explore the operating temperature-dependent PL performance of the phosphor, the Arrhenius equation (Eq. (S11) in the ESM) was employed for assessing the activation energy $\left(E_{\mathrm{a}}\right)$. Figure 10(c) shows the influence of $1 / k T$ on $\ln \left[\left(I_{0} / I(T)\right)-1\right]$. The $E_{\mathrm{a}}$ value of the KNBP: $0.75 \% \mathrm{Sm}^{3+}$ powder is around $0.31 \mathrm{eV}$ via fitting the experimental data. The synthesized phosphor has good thermostability comparing with reported inorganic phosphors doped with $\mathrm{Sm}^{3+}$, such as $\mathrm{SrBi}_{2} \mathrm{Ta}_{2} \mathrm{O}_{9}: \mathrm{Sm}^{3+}$ $\left(E_{\mathrm{a}}=0.23 \mathrm{eV}\right)$ [27], $\mathrm{Ca}_{19} \mathrm{Mg}_{2}\left(\mathrm{PO}_{4}\right)_{14}: \mathrm{Sm}^{3+}\left(E_{\mathrm{a}}=0.13 \mathrm{eV}\right)$ [28], and $\mathrm{KLaSr}_{3}\left(\mathrm{PO}_{4}\right)_{3} \mathrm{~F}: \mathrm{Sm}^{3+}\left(E_{\mathrm{a}}=0.163 \mathrm{eV}\right)$ [29]. Equation (S12) in the ESM was employed for analyzing color stability. The obtained parameter of chromaticity shift $(\Delta E)$ is around 0.029 at $503 \mathrm{~K}$, which is smaller than $\Delta E=0.044$ at $500 \mathrm{~K}$ for commercial red $\mathrm{CaAlSiN}_{3}: \mathrm{Eu}^{2+}$ product [30].

\section{Conclusions}

In conclusion, a new alkali metal borophosphate-based orange-red phosphor KNBP: $\mathrm{Sm}^{3+}$ was synthesized via a facile solid-state synthesis procedure. Despite dopant $\mathrm{Sm}^{3+}$ occupied the $\mathrm{Na}^{+}$site, the prepared KNBP: $x \mathrm{Sm}^{3+}$ $(x=0,0.25,0.5,0.75,1,2,3 \mathrm{~mol} \%)$ microparticles keep the orthorhombic structure of the KNBP matrix. The phosphor exhibits the typical $4 \mathrm{f}-4 \mathrm{f}$ transitions of $\mathrm{Sm}^{3+}$ at $645 \mathrm{~nm}\left({ }^{4} \mathrm{G}_{5 / 2} \rightarrow{ }^{6} \mathrm{H}_{9 / 2}\right), 598\left({ }^{4} \mathrm{G}_{5 / 2} \rightarrow{ }^{6} \mathrm{H}_{7 / 2}\right)$, and $562\left({ }^{4} \mathrm{G}_{5 / 2} \rightarrow{ }^{6} \mathrm{H}_{5 / 2}\right)$ upon $402 \mathrm{~nm}$ NUV irradiation. For the concentration quenching with the optimal $0.75 \mathrm{~mol} \%$ concentration of $\mathrm{Sm}^{3+}$, the electric dipole-dipole interaction plays a dominant role. The operating temperature-dependent photoluminescence reveals that the phosphor has good thermostability with the activation energy of $0.31 \mathrm{eV}$. The obtained properties confirm that the KNBP: $\mathrm{Sm}^{3+}$ phosphor with high $\mathrm{CP}$ and low CCT can potentially serve solid-state lighting and display applications.

\section{Acknowledgements}

This study is financially supported by the National Natural Science Foundation of China (Nos. 21761034 and 22165031), the Open Foundation of Key Laboratory for Micro/Nano Materials \& Technology of Yunnan Province (No. 2021KF01), and the Program for Excellent Young Talents, Yunnan University. We thank Advanced Analysis and Measurement Center of Yunnan University for the sample testing service.
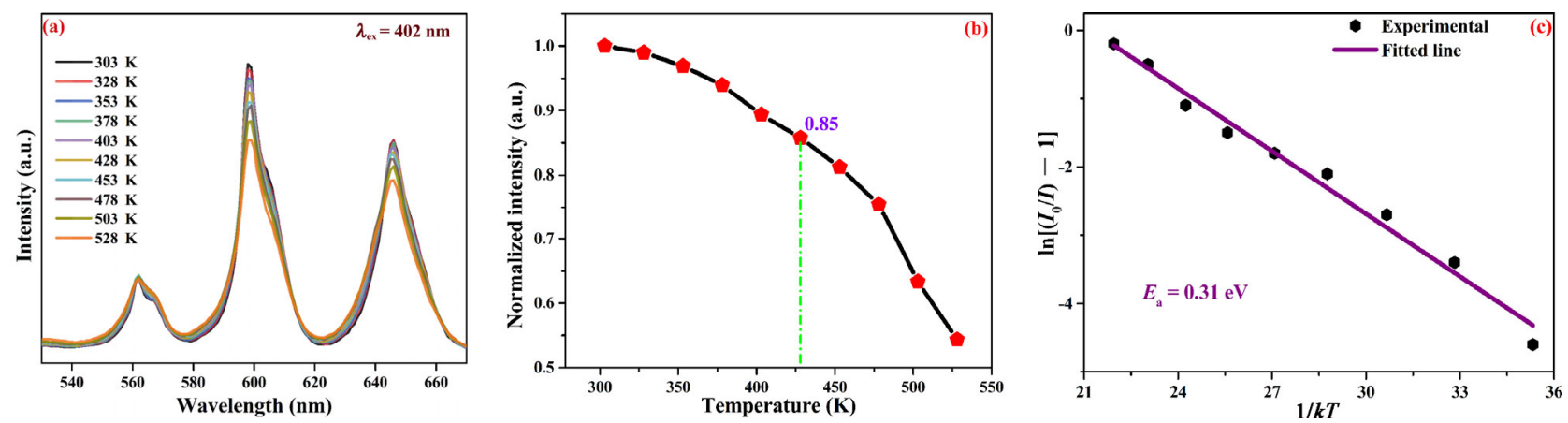

Fig. 10 (a) Operating temperature-dependent PL curves excited by a $402 \mathrm{~nm}$ light, (b) normalized emission intensities depending on different temperatures, and (c) activation energy of the thermal quenching of the KNBP:0.75\% $\% \mathrm{Sm}^{3+}$ powder. 


\section{Electronic Supplementary Material}

Supplementary material is available in the online version of this article at https://doi.org/10.1007/s40145-021-0492-z.

\section{References}

[1] Fang MH, Leaño JL Jr, Liu RS. Control of narrow-band emission in phosphor materials for application in lightemitting diodes. ACS Energy Lett 2018, 3: 2573-2586.

[2] Qin X, Liu XW, Huang W, et al. Lanthanide-activated phosphors based on $4 \mathrm{f}-5 \mathrm{~d}$ optical transitions: Theoretical and experimental aspects. Chem Rev 2017, 117: 4488-4527.

[3] Xia ZG, Liu QL. Progress in discovery and structural design of color conversion phosphors for LEDs. Prog Mater Sci 2016, 84: 59-117.

[4] Qiao JW, Zhao J, Liu QL, et al. Recent advances in solidstate LED phosphors with thermally stable luminescence. J Rare Earths 2019, 37: 565-572.

[5] Sahoo S. Enhanced time response and temperature sensing behavior of thermistor using $\mathrm{Zn}$-doped $\mathrm{CaTiO}_{3}$ nanoparticles. $J$ Adv Ceram 2018, 7: 99-108.

[6] Zhu Q, Wang XJ, Li JG. Recent progress in layered rare-earth hydroxide (LRH) and its application in luminescence. $J$ Adv Ceram 2017, 6: 177-186.

[7] Ofelt GS. Intensities of crystal spectra of rare-earth ions. $J$ Chem Phys 1962, 37: 511-520.

[8] Yang Y, Pan H, Guan L, et al. Electronic structure and luminescent properties of $\mathrm{Sr}_{3} \mathrm{Al}_{2} \mathrm{O}_{6}: \mathrm{Sm}^{3+}$ orange phosphor prepared by hydrothermal method. J Mater Res Technol 2020, 9: 3847-3855.

[9] Puchalska M, Zych E. The effect of charge compensation through alkali metal co-doping on the luminescence behaviour of $\mathrm{SrAl}_{4} \mathrm{O}_{7}: \mathrm{Sm}^{3+}$ phosphor. J Lumin 2018, 197: 211-218.

[10] Xin SY, Zhou FG, Wang C, et al. Thermal stable red phosphor $\mathrm{Sm}^{3+}$ doped $\mathrm{Na}_{3} \mathrm{MgZr}\left(\mathrm{PO}_{4}\right)_{3}$ : The synthesis, site occupation and photoluminescence property investigation. J Mater Sci: Mater Electron 2017, 28: 19134-19138.

[11] Fan J, Zhang WT, Dai SY, et al. Effect of charge compensators $\mathrm{A}^{+}(\mathrm{A}=\mathrm{Li}, \mathrm{Na}$ and $\mathrm{K})$ on luminescence enhancement of $\mathrm{Ca}_{3} \mathrm{Sr}_{3}\left(\mathrm{PO}_{4}\right)_{4}: \mathrm{Sm}^{3+}$ orange-red phosphors. Ceram Int 2018, 44: 20028-20033.

[12] Li T, Wang ZJ, Li PL, et al. A novel red emitting phosphor $\mathrm{LiBaB}_{9} \mathrm{O}_{15}: \mathrm{Sm}^{2+} / \mathrm{Sm}^{3+}, \mathrm{Li}^{+}$with broad excitation band for white LEDs. Luminescence 2018, 33: 438-442.

[13] Devi LL, Jayasankar CK. Novel reddish-orange color emitting $\mathrm{Ca}_{2} \mathrm{SiO}_{4}: \mathrm{Sm}^{3+}$ phosphors for white LED applications prepared by using agricultural waste. J Lumin 2020, 221: 116996.

[14] Han B, Dai YZ, Zhang J, et al. Photoluminescence properties of a double perovskite tungstate based redemitting phosphor $\mathrm{NaLaMgWO}_{6}: \mathrm{Sm}^{3+}$. Ceram Int 2018, 44: 3734-3740.
[15] Du P, Hua YB, Yu JS. Energy transfer from $\mathrm{VO}_{4}{ }^{3-}$ group to $\mathrm{Sm}^{3+}$ ions in $\mathrm{Ba}_{3}\left(\mathrm{VO}_{4}\right)_{2}: 3 x \mathrm{Sm}^{3+}$ microparticles: A bifunctional platform for simultaneous optical thermometer and safety sign. Chem Eng J 2018, 352: 352-359.

[16] Xie JH, Zhang XY, Cheng LQ, et al. Photoluminescence properties of novel $\mathrm{K}_{2} \mathrm{Mg}_{2}\left(\mathrm{MoO}_{4}\right)_{3}: \mathrm{Sm}^{3+}$ orange-red emitting phosphors for white light emitting diodes. Opt Mater 2020, 107: 110008.

[17] Li M, Verena-Mudring A. New developments in the synthesis, structure, and applications of borophosphates and metalloborophosphates. Cryst Growth Des 2016, 16: 2441-2458.

[18] Yang Y, Gong PF, Huang Q, et al. $\mathrm{KNa}_{4} \mathrm{~B}_{2} \mathrm{P}_{3} \mathrm{O}_{13}$ : A deep-ultraviolet transparent borophosphate exhibiting second-harmonic generation response. Inorg Chem 2019, 58: 8918-8921.

[19] Shannon RD. Revised effective ionic radii and systematic studies of interatomic distances in halides and chalcogenides. Acta Crystallogr Sect A 1976, 32: 751-767.

[20] Zhu J, Yang MH, Che Y, et al. Europium (III) doped $\mathrm{LiNa}_{2} \mathrm{~B}_{5} \mathrm{P}_{2} \mathrm{O}_{14}$ phosphor: Surface analysis, DFT calculations and luminescent properties. $J$ Alloys Compd 2020, 822: 153606.

[21] Zhou Y, Ge XC, Zhang ZH, et al. Understanding the downconversion photoluminescence of $\mathrm{NaLaP}_{4} \mathrm{O}_{12}: \mathrm{Er}^{3+}$ phosphor. Ceram Int 2019, 45: 20988-20993.

[22] Zhang $\mathrm{ZJ}, \mathrm{Hu} \mathrm{GQ}$, Wang $\mathrm{H}$, et al. Investigations of $\mathrm{Ba}_{3} \mathrm{BP}_{3} \mathrm{O}_{12}: \mathrm{Eu}^{2+}$ single crystal as a scintillator. Mater Res Bull 2014, 52: 70-73.

[23] Xiang JY, Yang MH, Che Y, et al. Photoluminescence investigation of novel reddish-orange phosphor $\mathrm{Li}_{2} \mathrm{NaBP}_{2} \mathrm{O}_{8}: \mathrm{Sm}^{3+}$ with high $\mathrm{CP}$ and low CCT. Ceram Int 2019, 45: 7018-7024.

[24] Zhao D, Shi LY, Zhang RJ, et al. Synthesis, crystal structure and luminescence properties of a new samarium borate phosphate, $\mathrm{CsNa}_{2} \mathrm{Sm}_{2}\left(\mathrm{BO}_{3}\right)\left(\mathrm{PO}_{4}\right)_{2}$. Acta Crystallogr Sect C 2020, 76: 1068-1075.

[25] Hooda A, Khatkar SP, Khatkar A, et al. Reddish-orange light emission via combustion synthesized $\mathrm{Ba}_{3} \mathrm{Y}_{4} \mathrm{O}_{9}: \mathrm{Sm}^{3+}$ nanocrystalline phosphor upon near ultraviolet excitation. $J$ Lumin 2020, 217: 116806.

[26] Li JH, Liang QY, Cao YF, et al. Layered structure produced nonconcentration quenching in a novel $\mathrm{Eu}^{3+}$-doped phosphor. ACS Appl Mater Interfaces 2018, 10: 4147941486.

[27] Zhong YF, Deng B, Gao X, et al. High thermally $\mathrm{Sm}^{3+}$ activated $\mathrm{SrBi}_{2} \mathrm{Ta}_{2} \mathrm{O}_{9}$ orange-red phosphor: Preparation, characterization, and optical properties. J Lumin 2019, 215: 116648.

[28] Zhu G, Ci ZP, Shi YR, et al. Synthesis and photoluminescence properties of $\mathrm{Ca}_{19} \mathrm{Mg}_{2}\left(\mathrm{PO}_{4}\right)_{14}: \mathrm{Sm}^{3+}$ red phosphor for white light emitting diodes. Mater Res Bull 2014, 55: 146-149.

[29] Guo QF, Zhao CL, Liao LB, et al. Luminescence investigations of novel orange-red fluorapatite $\mathrm{KLaSr}_{3}\left(\mathrm{PO}_{4}\right)_{3} \mathrm{~F}: \mathrm{Sm}^{3+}$ 
phosphors with high thermal stability. $J$ Am Ceram Soc 2017, 100: 2221-2231.

[30] Huang L, Zhu YW, Zhang XJ, et al. HF-free hydrothermal route for synthesis of highly efficient narrow-band red emitting phosphor $\mathrm{K}_{2} \mathrm{Si}_{1-x} \mathrm{~F}_{6}: x \mathrm{Mn}^{4+}$ for warm white lightemitting diodes. Chem Mater 2016, 28: 1495-1502.

Open Access This article is licensed under a Creative Commons Attribution 4.0 International License, which permits use, sharing, adaptation, distribution and reproduction in any medium or format, as long as you give appropriate credit to the original author(s) and the source, provide a link to the Creative Commons licence, and indicate if changes were made.

The images or other third party material in this article are included in the article's Creative Commons licence, unless indicated otherwise in a credit line to the material. If material is not included in the article's Creative Commons licence and your intended use is not permitted by statutory regulation or exceeds the permitted use, you will need to obtain permission directly from the copyright holder.

To view a copy of this licence, visit http://creativecommons. org/licenses/by/4.0/. 